Progress Report for FY 1992 of the research program

funded by DOE grant DE-FG03-90ER40546

DOE/ER/40546--T2

DE93 000649

\title{
THEORETICAL PARTICLE PHYSICS
}

\section{University of California, San Diego}

September 30, 1992

\section{DISCLAIMER}

\footnotetext{
This report was prepared as an account of work sponsored by an agency of the United States Government. Neither the United States Government nor any agency thereof, nor any of their employees, makes any warranty, express or implied, or assumes any legal liability or responsibility for the accuracy, completeness, or usefulness of any information, apparatus, product, or process disclosed, or represents that its use would not infringe privately owned rights. Reference herein to any specific commercial product, process, or service by trade name, trademark, manufacturer, or otherwise does not necessarily constitute or imply its endorsement, recommendation, or favoring by the United States Government or any agency thereof. The views and opinions of authors expressed herein do not necessarily state or reflect those of the United States Government or any agency thereof.
}

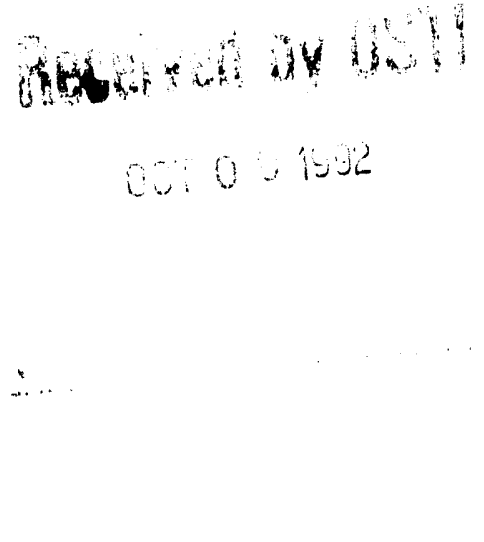




\section{TABLE OF CONTENTS}

1. Research Program . . . . . . . . . . . . . . . . 2

A. Quantum Chromodynamics . . . . . . . . . . 2

1. Heavy Quark Physics. . . . . . . . . . . . . . . . 2

2. Chiral Perturbation Theory. . . . . . . . . . 3

3. Skyrmions . . . . . . . . . . . . . . . . . . 3

4. Quarkonia and Nuclear Matter . . . . . . . . . . 4

5. Parity Violating Nuclear Matrix Elements . . . . . . 4

6. How Precisely can one Determine $M_{U} / M_{D}$ ? . . . . . . 4

B. Electroweak Interactions . . . . . . . . . . . . . . 6

1. Weak Scale Baryogenesis . . . . . . . . . . . . . . 6

2. Constraints of Baryogenesis from Neutrino Masses . . 8

3. Majorons, Double Beta Decay, Supernova 1987A . . . 8

4. Rare Decays . . . . . . . . . . . . . . . . . . . 9

C. Lattice Field Theory . . . . . . . . . . . . . . . 10

1. Chiral Lattice Fermions . . . . . . . . . . . . . . 10

2. Pauli-Villars Regulator and the Higgs Mass Bound . . 10

3. Higgs and Yukawa Interactions . . . . . . . . . . . 11

2. Contract Publications . . . . . . . . . . . . . . . . 12

3. Conference Talks . . . . . . . . . . . . . . . 15 


\section{[1] RESEARCH PROGRAM}

\section{A. QUANTUM CHROMODYNAMICS}

\section{HEAVY QUARK PHYSICS}

(Elizabeth Jenkins, Michael Luke, Aneesh Manohar, Martin Savage)

A significant component of the group's research program in the past year has been in the field of QCD phenomenology, with emphasis on the phenomenology of mesons and baryons containing a single heavy ( $c$ or $b$ ) quark. In the limit in which the mass of the heavy quark becomes infinite, the dynamics of these particles simplifies dramatically, and the resulting "heavy quark effective theory" (HQET) has spin and flavour symmetries not manifest in the original QCD lagrangian. With $A$. Falk and M. Neubert (UCSD/PTH 92-09) Luke has discussed the technical issue of how to precisely and unambiguously define the (renormalization prescription dependent) heavy quark mass which occurs in defining the HQET, and shown that physical observables are independent of the prescription used to define the mass. Similarly, the velocity of the heavy quark is another seemingly ambiguous parameter which occurs in the HQET; Luke and A. Manohar (Phys. Lett. B286 (1992) 348) have shown that not only are physical observables independent of the choice of velocity, but that invariance under choice of velocity places strong constraints on the types of operators which may arise in the theory, placing non-perturbative restrictions on the coefficients of certain operators in the HQET.

The heavy quark linit can also be used to study systems with two heavy quarks, such as the $B_{c}$ meson. The present run at Fermilab is expected to produce around 50 identified $B_{c}$ events. The heavy quark symmetry determines the decay form factor for $B_{c}$ semileptonic decay in terms of the $B$ meson decay constant $f_{B}$. This allows the weak mixing angles in $b$ quark decay to be measured with no normalization uncertainty. These consequences were worked out by $M$. Luke, E. Jenkins, A. Manohar and M. Savage (UCSD/PTH 92-13).

There has been much recent interest in the synthesis of heavy quark symmetry and the chiral $S U(3)_{L} \times S U(3)_{R}$ symmetry of the three light quark flavours. By incorporating the heavy hadrons into a chiral lagrangian one can describe the interactions of heavy hadrons with soft pions, kaons and etas. With A. Falk (UCSD/PTH 92-14), Luke examined the strong decays of excited $p$-wave $D$ mesons $\left(D_{2}^{*}(2460)\right.$ and $\left.D_{1}(2420)\right)$ to the ground state $D$ and $D^{*}$ mesons and one or two pions, while J.F. Amundson, C.G. Boyd, J.L. Rosner, M. Luke, A.V. Manohar, 
M.J. Savage and M.B. Wise (UCSD/PTH 92-31) examined SU(3) breaking in radiative $D^{*} \rightarrow D \gamma$ decays and determined the $D \pi D^{*}$ coupling constant, allowing them to predict the absolute width of the $D^{*}$ meson.

\section{CHIRAL PERTURBATION THEORY}

(Elizabeth Jenkins, Michael Luke, Aneesh Manohar, Martin Savage)

Chiral corrections to mesons containing a heavy quark can be computed by combining standard methods of chiral perturbation theory and heavy quark symmetry. This was applied to determine the leading non-analytic corrections to the meson decay constants for the $D$ mesons, $f_{D}$, and to the parameter $B_{B}$ in $B-\bar{B}$ mixing.

E. Jenkins calculated leading non-analytic corrections to hyperon nonleptonic decay amplitudes in chiral perturbation theory. Success of the Lee-Sugawara relation for S-wave amplitudes is understood in the context of chiral perturbation theory. P-wave amplitudes are not well-described by one-loop chiral perturbation theory since the tree-level $S U(3)$ symmetric contribution to these amplitudes is significantly suppressed. In addition, the conventional definition of $\mathrm{P}$-wave amplitudes is replaced by a new definition which naturally arises in the heavy baryon formalism and has a legitimate $1 / m_{B}$ limit, where $m_{B}$ is the chiral invariant mass of the baryon multiplet.

With Malcolm Butler, Savage has been investigating the polarisability of the nucleon in the framework of chiral perturbation theory. They found that the isovector polarisability was determined by kaon loops at leading order and hence strange-quark configurations of the nucleon. Also they included the decuplet of resonances and found them to be significant. This has lead them to investigate the two photon decay of the $\Sigma^{0}$, this is work still in progress.

\section{SKYRMIONS}

\section{(Michael Luke and Aneesh Manohar)}

It has long been known that light baryons may be described as solitons in an $S U(3)_{L} \times S U(3)_{R}$ chiral lagrangian (the "Skyrme model"); this description corresponds to the large $N_{c}$ limit of QCD. M. Luke, A. Manohar and Z. Guralnik (UCSD/PTH 92-24) examined the corresponding picture of heavy baryons, in which they are treated as bound states of a heavy meson and a soliton. In this framework, they calculated the masses of the $\Lambda_{c}$ and $\Sigma_{c}$ baryons and found the resu'ts to igree well with experiment. They also calculated the $\Sigma_{c} \Sigma_{c} \pi$ and $\Sigma_{c} \Lambda_{c} \pi$ coupling constants, allowing them to predict the width of the $\Sigma_{c}$ which has not yet been measured experimentally. They also found that exotic bound states with no analogue in the quark model which could arise in this model were unbound. 


\section{QUARKONIA AND NUCLEAR MATTER}

\section{(Michael Luke, Aneesh Manohar, Martin Savage)}

Luke, Manohar, and Sarage realised that the interaction of quarkonia with nuclear matter (and finite nuclei) could be described perturbatively in QCD by exploiting a multipole expansion that exists when the radius of the quarkonia is small compared with the hadronic scale. Heavy quark spin symmetry allows them to put the pseudoscalar and vector quarkonia states in a spin multiplet and show that the spin-spin and spin-orbit interactions are suppressed by $\alpha_{s}\left(r_{Q}\right)$ compared with the leading interaction. Low energy theorems of $\mathrm{QCD}$, scale invariance and the known matrix element of the twist-2 gluon operator $\theta_{\mu \nu}$ allowed them to determine the binding energy of quarkonia to nuclear matter. They found this binding energy to be very small, of order $10 \mathrm{MeV}$ for the $J / \psi$ and of order a few $\mathrm{MeV}$ for the $\Upsilon$.

\section{PARITY VIOLATING NUCLEAR MATRIX ELEMENTS}

(David Kaplan and Martin Savage)

Kaplan and Savage examined the role of QCD symmetries manifested in Chiral Perturbation theory have on the form of parity violating operators relevant for low energy nuclear physics observables. They found many more operators than had been discussed previously and provided a possible solution to the conflict between data sets extracted from measurements on light nuclei of parity violating couplings.

\section{HOW PRECISELY CAN ONE DETERMINE $M_{U} / M_{D}$ ?}

\section{(Kiwoon Choi)}

A rather precise knowledge of the light quark masses may be essential for a better understanding of the entire quark mass spectrum and the weak mixing angles. Also the possibility for $m_{u}=0$ is particularly interesting in connection with the strong CP problem. It has been well known that due to the instanton induced light quark mass renormalization there exists an intrinsic ambiguity in determining the quark mass ratios, particularly $m_{u} / m_{d}$, within chiral perturbation theory. The reason for the ambiguity is that the instanton induced mass can not be distinguished from the bare mass in chiral perturbation theory. In order to resolve this ambiguity, one needs to do the QCD calculation of some second order chiral coupling constants, e.g. $L_{7}$ in the chiral lagrangian of mesons. Recently the QCD spectral function representation of $L_{7}$ was derived by Leutwyler. In this spectral representation, $S U(3)$ singlet pseudoscalar states give negative contributions to $L_{7}$ while $S U(3)$ octet pseudoscalar states (excluding $\pi, K$ and $\eta$ ) give positive 
ones. If $L_{7}$ is negative, the corresponding value of $m_{u} / m_{d}$ would be securely away from zero. Leutwyler then argued that $m_{u}$ can not be zero since it is likely that $L_{7}$ is dominated by the negative contribution from the lowest available state $\eta^{\prime}$. After this claim, Choi investigated how QCD instantons affect the chiral coupling constant $L_{7}$. He found that instantons suppress the negative $\eta^{\prime}$ contribution while enhancing the positive contribution from $S U(3)$ octet pseudoscalar resonances. As a result, the major contribution to $L_{7}$ can come from the octet resonances, not from the singlet $\eta^{\prime}$. This means that $L_{7}$ can be positive, allowing $m_{u}=0$ to be an open possibility. 


\section{B. ELECTROWEAK INTERACTIONS}

\section{WEAK SCALE BARYOGENESIS}

\section{(Ann Nelson and David Kaplan)}

Kaplan and Nelson have continued studying the possibility of baryogenesis from anomalous weak interactions during the weak phase transition. New results include a computation of the baryon asymmetry of the universe prodicted in the two Higgs doublet model, as a function of parameters associated with the weak transition and CP violating phases. They found that this model can easily produce the observed baryon abundance for $\mathrm{CP}$ violating phases in the scalar potential as small as $10^{-4}$. The mechanism for converting $C P$ violation into baryon number involves the asymmetric reflection probabilities between left and right handed top quarks, which produces a net top quark hypercharge in the symmetric phase. The effects of Debye screening on this hypercharge prevent a large hypercharge from building up in the symmetric phase, but they have shown that this screening converts hypercharge into baryon number. Thus the phase boundary ends up reflecting net (anti)baryon number into the symmetric phase. Anomalous baryon violating transitions are unsuppressed in the symmetric phase, and will tend to bring the system towards thermal equilibrium by creating net baryon number.

The detailed computation of the baryon asymmetry proceeds as follows ${ }^{\dagger}$ :

Step 1. First compute the probability that a top quark of definite chirality and momentum incident on the bubble wall is reflected. The reason to focus on the top quark is that there is a significant probability for both reflection and transmission of top quarks off of the bubble wall, and so $\mathrm{CP}$ violating effects of the reflection coefficients are large.

Step 2. The result of step 1 is integrated against thermal distributions of quarks inside and outside the bubble to give the net axial top quark flux emanating from the bubble wall. This flux includes quarks reflected from the bubble wall as well as quarks transmitted from the bubble interior into the false vacuum region. Using unitarity and $C P T$, the probability for transmission of quarks from the interior of the bubble may be related to the reflection coefficients computed in step 1.

$\dagger$ A. E. Nelson, D. B. Kaplan, and A. G. Cohen, "Why there is Something Rather than Nothing: Matter from Weak Interactions", Nuclear Physics B373 (1992) 453. A. G. Cohen, D. B. Kaplan, and A. E. Nelson, "Debye screening and baryogenesis during the electroweak phase transition", UCSD/PTH 92-19, accepted for publication in Physics Letters B. 
The total flux will of course go to zero as the wall velocity $u$ goes to zero; in this case the transmitted flux exactly cancels the reflected flux. As an example of the magnitudes involved, for a wall velocity of $u=1 / \sqrt{3}$ that the flux in units of the photon density at the critical temperature $T$ could be as large as 0.1 for maximal $C P$ violation, $m_{t} \simeq 2 T$, and wall width of order $1 / T$.

Step 3. The flux of hypercharge into the false vacuum region is affected by Debye screening, since a long range hypercharge field cannot exist in the symmetric phase. The particle distribution functions in the plasma approximately satisfy the linearized Maxwell-Boltzmann equations of

$$
\begin{aligned}
& v_{z} \frac{\partial f_{i}}{\partial z}+q_{i} E \frac{\partial f_{0, i}}{\partial p_{z}}=I_{i} \\
& \frac{\partial E}{\partial z}=\sum_{i} q_{i} \int f_{i} d^{3} p .
\end{aligned}
$$

Here the equilibrium distribution for the $i^{\text {th }}$ species are denoted as $f_{0, i}(\vec{p}, \vec{x})$, and the departure from equilibrium as $f_{i}(\vec{p}, \vec{x})$. The collision term, $I_{i}$, represents the effects of interactions in the system, and vanishes for all distributions equal to their equilibrium values. $q_{i}$ is the hypercharge of particle species $i$, the particle distribution functions are taken to be time independent, and $E$ is the hypercharge electric field, which points in the $z$ direction. The effect of the term $q_{i} E \frac{\partial f_{0, i}}{\partial p_{z}}$ can be shown to convert the reflected hypercharge into net (anti)baryon number, which causes a bias in anomalous weak interactions favoring the production of baryon number over anti-baryon number. Finding the particle distribution functions necessitates solving the Boltzmann equations, a complicated task beyond the scope of this paper. Instead they performed a simplified computation, ignoring all interactions except top quark scattering off of other quarks via gluon exchange (numerically the most important inceraction). They then performed a Monte Carlo simulation of the propagation of the axial top quark flux computed in step 2 through the hot plasma in front of the bubble wall. This computation leads to a net hypercharge extending in front of the wall over distances of up to $\mathcal{O}(100) / T$. They then used an argument that Debye screening of this hypercharge field would not significantly affect global charges which are orthogonal to hypercharge to compute the densities of various global quantum numbers (such as baryon number) which would be induced by screening.

Step 4. Assuming that the system is close to equilibrium, they solved for the local chemical potentials which would result in the charge densities computed in step 3 , and then computed the rate of baryon production with the rate equation

$$
\dot{\rho}_{B}=-\frac{\Gamma_{B}}{T} \frac{\partial F}{\partial B}
$$


where the derivative of the free energy with respect to the baryon number is computed with all quantum numbers which are not violated by anomalous weak interactions held fixed.

\section{CONSTRAINTS OF BARYOGENESIS FROM NEUTRINO MASSES}

\section{(Ann Nelson)}

Nelson * showed that most particle physics models consistent with the 17 $\mathrm{keV}$ neutrino require a baryogenesis scale below $\sim 10^{6} \mathrm{GeV}$. Furthermore models with a $17 \mathrm{keV}$ neutrino typically contain new sources of CP violation, and the cosmological baryon asymmetry could be generated by anomalous electroweak interactions during a first order weak phase transition, via a similar mechanism to the one detailed below.

\section{MAJORONS, DOUBLE BETA DECAY, SUPERNOVA 1987 A}

\section{(Kiwoon Choi)}

Models with spontaneous lepton number violation can have many interesting phenomenological consequences associated with the accompanying Goldstone boson "majoron". Recently it has been pointed out that the potential anomaly in the double beta decay spectra of several elements may be explained by the neutrinoless emission of majorons. Independently of this, the majoron Yukawa coupling $g$ with $\nu_{e}$ in the range $10^{-5}$ to $10^{-3}$ is interesting in connection with the future possibility for observable "neutrinoless double beta decay with majoron emission" $\left(\beta \beta_{J}\right)$. Sometime ago, Kolb and Turner considered the interactions of supernova neutrinos with cosmic background majorons (and also $\nu_{e}$ 's). They then obtained $g \leq 10^{-3}$ from the requirement that the neutrino mean free path must be comparable to or greater than the distance to the supernova 1987A. However Kolb and Turner's analysis was performed in the limit where the scalar quartic coupling constant $\lambda$ in the Higgs potential is vanishingly small. Chang and Choi reanalyzed this problem for generic values of $\lambda$ and found that some part of the parameter region leading to observable $\beta \beta_{J}$ by giving $g=10^{-5} \sim 10^{-3}$ can be excluded by the neutrino pulse from the supernova $1987 \mathrm{~A}$.

* A. E. Nelson, "Implications of a $17 \mathrm{KeV}$ neutrino for baryogenesis", UCSD/PTH 91-29, accepted for publication in Nuclear Physics B. 


\section{RARE DECAYS}

(Michael Luke and Martin Savage)

Mark Wise, Ming-Lu and Martin Savage examined the contribution of twophoton intermediate states to the parity polarisation asymmetry of the muon polarisation in the rare decay $K^{+} \rightarrow \pi^{+} \mu^{+} \mu^{-}$. CPS symmetry forced one of the parity violating contact terms to vanish at lowest order in chiral perturbation theory and we computed the leading contribution arising from a $\pi^{0} / \eta$ intermediate state. They found that this contribution was small compared with that from short-distance physics from top and charm loops when $\rho<0$ in the Wolfenstein parametrisation when $V_{b c}$ was real. This gave them confidence (as they could not compute the form factor that did not vanish due to CPS), that the long-distance two-photon amplitudes were not significant for large $\operatorname{Re}\left(V_{t d}\right)$. As a spin off of this computation, Luke and Savage were able to predict the rate for $\pi^{0} \rightarrow e^{+} e^{-}$and $\eta \rightarrow e^{+} e^{-}$from the recently measured $\eta \rightarrow \mu^{+} \mu^{-}$. 


\section{LATTICE FIELD THEORY}

\section{CHIRAL LATTICE FERMIONS}

(Karl Jansen and David Kaplar.)

The most recent works by Jansen are related to the proposal by Kaplan for simulating chiral fermions on the lattice. He was able to show that the chiral zeromodes found on the infinite lattice survive on the finite lattice. In addition he could demonstrate that in the presence of weak external gauge fields the continuum anomaly equation is realized even on the lattice. This surprising fact was explained by Jansen in collaboration with M. Golterman and D. Kaplan by showing that the divergence of the Chern-Simons current has the same form on the lattice as in the continuum. The calculation of the Chern-Simons current on the lattice uses the fact that the fermion propagator can be interpreted as a map from the torus $T^{d}$ to the sphere $S^{d}$ in $d$-dimensions. The current gets only contributions where the winding number of this map changes. In collaboration with M. Schmaltz Jansen was able to show that this happens exactly at those points where the zeromode spectrum of the domain wall model changes.

\section{PAULI-VILLARS REGULATOR AND THE HIGGS MASS BOUND}

(Karl Jansen and Julius Kuti)

Jansen and Kuti proposed to investigate the physical properties of the $O(4)$ symmetric scalar field theory in four dimensions with Pauli-Villars regulated quartic self-interaction. The $\mathrm{O}(4)$ model plays a very important role in particle physics because it describes the symmetry breaking Higgs sector of the minimal Standard Model. A complex pair of Pauli-Villars regulator masses is introduced to render quantum fluctuations finite in a minimal fashion. When viewed as a finite field theory some excitations of the scalar $\mathrm{O}(4)$ field are quantized with negative metric in the Hilbert space. The complex pair of the heavy regulator masses corresponds to ghost particles which are expected to exibit unusual resonance properties. All symmetry properties of the model are otherwise preserved in the regularization process. At strong bare coupling the physical spectrum and the euclidean correlation functions of the model have to be determined from Monte Carlo simulation of the euclidean path integral. The main thrust of the research is to determine the Higgs mass bound before the unusual physical properties of the regulator particles begin to effect the low energy behavior of the minimal Standard Model. For computer simulation of the euclidean path integral one has to discretize the problem on a hypercubic lattice. The presence of the lattice parameter $a$ which can be 
tuned with respect to the regulator masses allows them to smoothly interpolate between the lattice regulated theory and the finite Pauli-Villars theory in the continuum. This is the first effort to determine the Higgs mass bound in the $O(4)$ model non-perturbatively without lattice regulator. Work is still in progress in this project. In the last six months Jansen, Kuti, and graduate student Liu have developed some detailed physical understanding of the higher derivative lagrangian field theory in the Higgs Sector and now they are in the process of investigating the model on the computer in a large scale simulation.

\section{HIGGS AND YUKAWA INTERACTIONS}

(Karl Jansen and Julius Kuti)

Together with $Y$. Shen research associate Jansen studied the tunneling energy in the 4-dimensional Ising model and extracted from its finite size behaviour the surface tension. They showed that this method is a well controlled and efficient way for getting the surface tension in lattice field theory.

K. Jansen, J. Kuti and Y. Shen addressed the question of a recent proposal that the scattering amplitude of W-bosons at the SSC might be structureless and one might therefore miss the Higgs sector. They clarified several misconceptions in existing literature concerning large- $\mathrm{N}$ expansion in the scalar field theory and pointed out that the appearance of tachyons in these theories has to be attributed to cut-off effects.

Jansen continued some earlier work on Yukawa-Higgs models on the lattice. With A. Hasenfratz and Y. Shen they studied for the first time these models for radially free Higgs fields and found surprising deviations from the case of fixed length Higgs fields which has been studied earlier. Jansen also looked at the finite temperature phase diagram of these models verifying the occurrence of the expected symmetry restoration. 


\section{[2] CONTRACT PUBLICATIONS}

months:

Contract publications are listed for the time period of the last twelve

1. A.E. Nelson, D.B. Kaplan, A.G. Cohen, Why there is Something Rather than Nothing: Matter from Weak Interactions, Nuclear Physics B373, (1992) 453.

2. A.E. Nelson, Implications of a $17 \mathrm{KeV}$ neutrino for baryogenesis, UCSD/PTH 92-29, accepted for publication in Nuclear Physics B.

3. K. Choi, D.B. Kaplan, A.E. Nelson, Is CP a Gauge symmetry?, UCSD/PTH 92-11, submitted for publication in Nuclear Physics B.

4. A.G. Cohen, D.B. Kaplan, A.E. Nelson, Debye screening and baryogenesis during the electroweak phase transitions, UCSD/PTH 92-19, accepted for publication in Physics Letters B.

5. D.B. Kaplan, M.J. Savage, An Analysis of Parity Violating Pion Nucleon Couplings, submitted to Nuclear Physics A.

6. B. Grinstein, E. Jenkins, A.V. Manohar, M.J. Savage, M.B. Wise, Chiral Perturbation Theory for $f_{D_{s}} / f_{D}$ and $B_{B_{s}} / B_{B}$, Nuclear Physics B380, (1992) 369.

7. E. Jenkins, M.J. Savage, Light Quark Dependence of the Isgur-Wise Function, Physics Letters B281, (1992) 331.

8. M. Luke, A.V. Manohar, M.J. Savage, A QCD Calculation of the Interaction of Quarkonium with Nuclei, to appear in Physics letters B.

9. E. Jenkins, M. Luke, A.V. Manohar, M.J. Savage, Semileptonic $B_{C}$ Decay and Heavy Quark Spin Symmetry, submitted to Nuclear Physics B.

10. M. Lu, M.J. Savage, M.B. Wise, Two-Photon Contribution to $K \rightarrow \pi \mu \mu$, CALT-68-1798, UCSD/PTH 92-20, submitted to Phys. Review D.

11. M.J. Savage, M. Luke, M.B. Wise, The Rare Decays $\pi^{0} \rightarrow e^{+} E^{-}$, $\eta \rightarrow e^{+} e^{-}$and $\eta \rightarrow \mu^{+} \mu^{-}$in Chiral Perturbation Theory, UCSD/PTH 92-23, CALT-68-1803, to appear in Physics Letters B.

12. M.N. Butler, M.J. Savage, The Electromagnetic Polarisability of the Nucleon, UCSD/PTH 92-30, sumitted to Physics Letters B.

13. J.F. Amundson, C.G. Boyd, J.L. Rosner, M. Luke, A.V. Manohar, M.J. Savage, M.B. Wise, Radiative $D *$ Meson Decay Using Heavy Quark and Chiral Symmetry, UCSD/PTH 92-31, in preparation. 
14. M.N. Butler, M.J. Savage, Two Photon Decay of the $\Sigma^{0} ; \Sigma^{0} \rightarrow \Lambda \gamma \gamma$, in preparation.

15. E. Jenkins, Hyperon Nonleptonic Decays in Chiral Perturbation Theory, Nuclear Physics 375B, (1992) 561-581.

16. E. Jenkins, A.V. Manohar, Baryon Chiral Perturbation Theory, Proceedings of the workshop on Effective Field Theories of the Standard Model, World Scientific, 1992.

17. E. Jenkins, A.V. Manohar, The Sigma Term and $m_{s}^{3 / 2}$ Corrections to the Proton Mass, Physics Letters 281B, (1992) 336-340.

18. K. Choi, How precisely can one determine $m_{u} / m_{d}$ ?, UCSD/PTH 92-01, to appear in Nuclear Physics B.

19. K. Choi, Light Quark Masses and Quarkonjum Decays, UCSD/PTH 92-06, to appear in Physics Letters B.

20. S. Chang, K. Choi, Majorons from Double Beta Decay and Supernova 1987A, UCSD/PTH 92-25.

21. K. Jansen, Tachyons in $\sigma$-Models, UCSD/PTH 92-01.

22. K. Jansen, Y. Shen, Tunneling and Energy Splitting in Ising Mdoels, UCSD/PTH 92-02.

23. A. Hasenfratz, K. Jansen, Y. Shen, The Phase Diagram of a U(1) Higgs Yukawa Model at Finite $\lambda$, UCSD/PTH 92-08.

24. K. Jansen, Chiral Fermions and Anomalies on a Finite Lattice, UCSD/PTH 92-18, to appear in Physics Letters B.

25. K. Jansen, Finite Temperature Phase Diagram for a U(1) Higgs-Yukawa Model, UCSD 92-22.

26. M.F.L. Golterman, D. Kaplan, K. Jansen, Chern-Simons Currents and Chiral Fermions on the Lattice, UCSD/PTH 92-28.

27. K. Jansen, M. Schmaltz, Critical Momenta of Lattice Chiral Fermions, UCSD/PTH 92-29.

28. A. Fulk, M. Luke, M. Neubert, The Residual Mass Term in the Heavy Quark Effective Theory, SLAC-PUB-5771/UCSD-PTH 92-09, to appear in Nuclear Physics B.

29. A. Falk, M. Luke, Strong Decays of Excited Heavy Mesons in Chiral Perturbation Theory, UCSD/PTH 92-14, SLAC-PUB-5812, to appear in Physics Letters B.

30. M. Luke, A.V. Manohar, Reparameterisation Invariance Constraints on Heavy Particle Effective Field Theories, Physics Letters B286, (1992) 348. 
31. Z. Guralnik, M. Luke, A.V. Manohar, Properties of Baryons Containing a Heavy Quark in the Skyrme Model, UCSD/PTH 92-24, submitted to Nuclear Physics B.

32. J. Kuti, The generalized Nambu-Jona-Lasinio mechanism and the standard model, Proceedings of the workshop on Effective Field Theories of the Standard Model, Dobojókö, Hungary, August 22-26,1991, edited by U.G. Meissuer, World Scientific 329.

33. J. Kuti, The Nambu-Jona-Lasinio mechanism in the standard model, Nuclear Physcs B (Proc. Suppl.) 26, (1992) 234.

34. J. Kuti, Non-perturbative study of the Higgs and Yukawa Couplings in the minimal standard model, Proceedings of the International Workshop on Electroweak Symmetry Breaking, Hiroshima, Japan, November 12-15, 1991, Edited by J. Kodaira, Worla Scientific Publisher. 


\section{[3] CONFERENCE TALKS}

1. A. Nelson, "Off the Wall Baryogenesis from Anomalous Weak Interactions", given at the Johns Hopkins Workshop on Current Problems in Particle Theory, August 1991.

2. A. Nelson, "Constraints on neutrino mixing with a $17 \mathrm{keV}$ neutrino, or Can there be one Majorana $17 \mathrm{keV}$ neutrino?", given at the Berkeley Workshop on the $17 \mathrm{keV}$ neutrino question, December 1991.

3. A. Nelson, "Baryogenesis from anomalous weak interactions at the weak phase transition", given at the Yale Workshop on Baryon Number Violation at the Electroweak Scale, New Haven, March 1992.

4. A. Nelson, "The Weak Phase Transition and Baryogenesis", given at the ITP workshop on Cosmological Phase transitions, Santa Barbara, April, 1992.

5. A. Nelson, "Baryogenesis at the Electroweak Scale", given at the APS meeting in Washington, April 1992.

6. A. Nelson, "Is CP a Gauge symmetry?", given at the International Seminar Quarks 92, Zvenigorod, Russia, May 1992..

7. E. Jenkins, "Including the Spin $3 / 2$ Decuplet in the Baryon Chiral Lagrangian", given at the 1991 Santa Fe workshop.

8. E. Jenkins, "Baryon Chiral Perturbation Theory", given at workshop on "Effective Field Theories of the Standard Model", Dobogókő, Hungary, August 1991.

9. A. Manohar, talk given at the "Puzzles on the Electroweak Scale" conference in Warsaw, Poland; June 1991.

10. A. Manohar, talk given at workshop on "Effective Field Theories of the Standard Model", Dobogókö, Hungary, August 1991.

11. A. Manohar, talk given at the "Lake Louise Winter Institute" conference in Lake Louise, Canada, February 1992.

12. A. Manohar, talk given at the "Conference on Quantum Phase Transitions", ITP Santa Barbara, June 1992. 
13. M. Luke, "Heavy Quark Symmetry", given at the Gordon Conference "Particle Physics in the 90's" in Andover, New Hampshire, July 1992.

14. J. Kuti, "The generalized Nambu-Jona-Lasinio mechanism and the standard model", given at the "Proceedings of the workshop on Effective Field Theories of the Standard Model" in Dobogóko,, Hungary, August 22-26, 1991.

15. J. Kuti, "The Nambu-Jona-Lasinio mechanism in the standard model", invited talk at Lattice 91, Tsukuba, Japan, November 5-9, 1991.

16. J. Kuti, "Non-perturbative study of the Higgs and Yukawa Couplings in the minimal standard model", given at the "Proceedings of the International Workshop on Electroweak Symmetry Breaking", Hiroshima, Japan, November 12-15, 1991.

17. J. Kuti, "The fate of the triviality mass bound with higher derivative Lagrangian in the Higgs sector", talk given at Lattice 92, Amsterdam, September 15-19, 1992. 

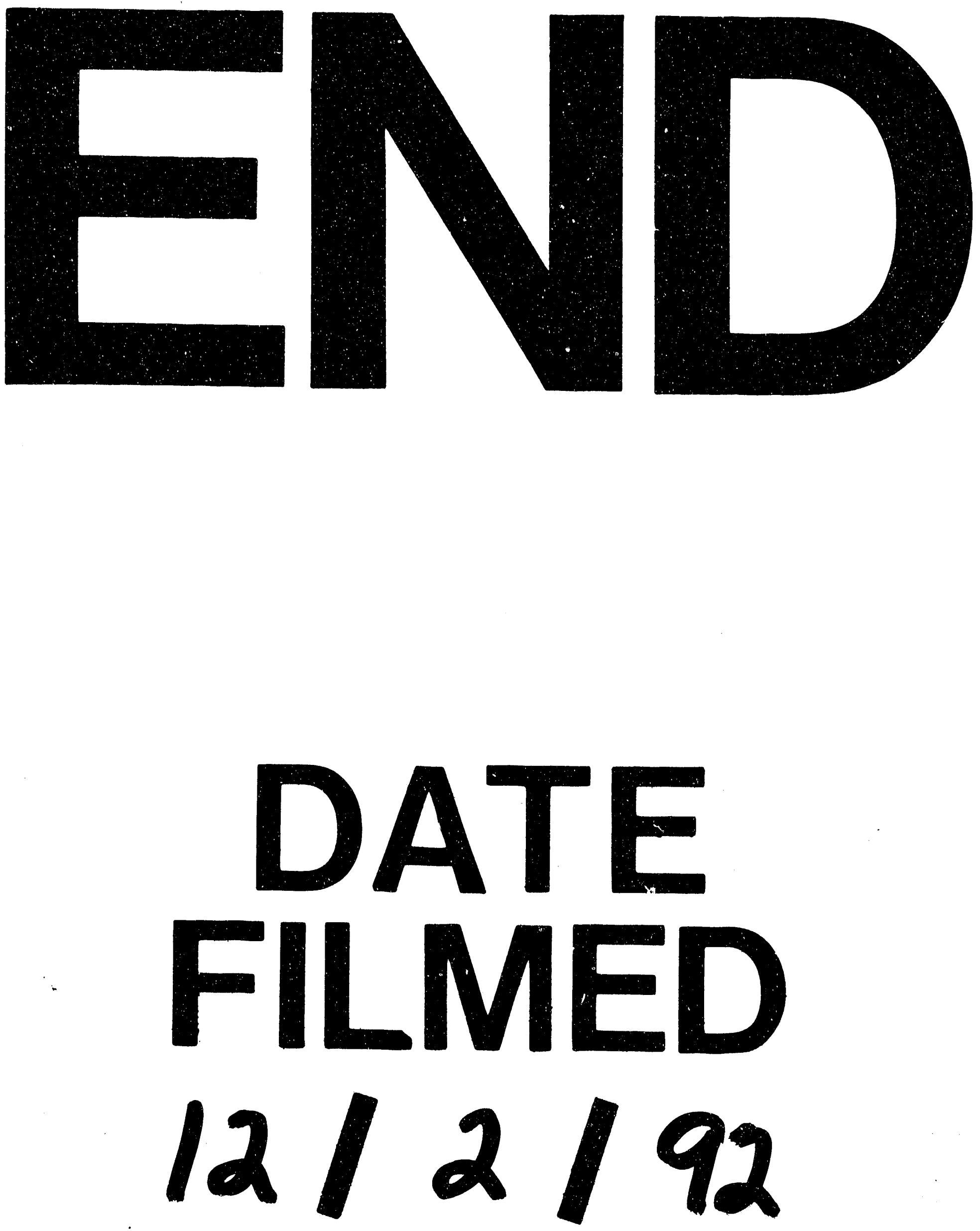
